\title{
ON FROBENIUS (COMPLETED) ORBIT CATEGORIES
}

\author{
ALFREDO NÁJERA CHÁVEZ
}

\begin{abstract}
Let $\mathcal{E}$ be a Frobenius category, $\mathcal{P}$ its subcategory of projective objects and $F: \mathcal{E} \rightarrow \mathcal{E}$ an exact automorphism. We prove that there is a fully faithful functor from the orbit category $\mathcal{E} / F$ into $\operatorname{gpr}(\mathcal{P} / F)$, the category of finitely-generated Gorensteinprojective modules over $\mathcal{P} / F$. We give sufficient conditions to ensure that the essential image of $\mathcal{E} / F$ is an extension-closed subcategory of $\operatorname{gpr}(\mathcal{P} / F)$. If $\mathcal{E}$ is in addition KrullSchmidt, we give sufficient conditions to ensure that the completed orbit category $\mathcal{E} \widehat{F}$ is a Krull-Schmidt Frobenius category. Finally, we apply our results on completed orbit categories to the context of Nakajima categories associated to Dynkin quivers and sketch applications to cluster algebras.
\end{abstract}

\section{Contents}

1. Introduction 1

Acknowledgments 3

2. Reminders on dg categories and their orbit categories 3

2.1. Pretriangulated dg categories. $\quad 3$

2.2. Quasi-functors.

2.3. Orbit categories. $\quad 5$

3. The ambient Frobenius category $\quad 8$

3.1. Frobenius categories and Gorenstein-projective modules 8

3.2. The ambient Frobenius category for the orbit category 9

4. Completed orbit categories $\quad 10$

5. Comparison of the triangulated structures $\quad 12$

6. The main theorems $\quad 14$

7. Applications to cluster algebras (sketch) 16

7.1. Nakajima categories $\quad 16$

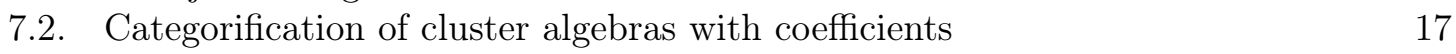

$\begin{array}{lr}\text { References } & 18\end{array}$

\section{INTRODUCTION}

Let $\mathcal{E}$ be an additive category and $F: \mathcal{E} \rightarrow \mathcal{E}$ an automorphism. The category of orbits associated to this data was first introduced by Cibils and Marcos in [9]. It was further studied by Asashiba in [1, 2] and by Keller in [21, 22]. By definition, the orbit category $\mathcal{E} / F$ has the same objects as $\mathcal{E}$, the set of morphisms from an object $X$ to an object $Y$ is given by

$$
\mathcal{E} / F(X, Y)=\bigoplus_{l \in \mathbb{Z}} \mathcal{E}\left(X, F^{l}(Y)\right)
$$

and the composition of morphisms is defined in a natural way (see (2.2)). Clearly $\mathcal{E} / F$ is still an additive category and the canonical projection $p: \mathcal{E} \rightarrow \mathcal{E} / F$ is an additive functor. 
Now suppose that $\mathcal{E}$ is a Frobenius category and that $F$ is an exact functor. We are interested in the following question:

Is there an exact structure on the orbit category such that $\mathcal{E} / F$ becomes a Frobenius category and the canonical projection an exact functor?

In this article we give sufficient conditions for the answer to be positive. Recall that Frobenius categories and triangulated categories are closely related: the stable category $\underline{\mathcal{E}}$ is canonically triangulated and $F$ induces a triangulated automorphism $\underline{F}$ on $\underline{\mathcal{E}}$. Moreover, the analogous question for triangulated categories was already studied in [21] where the author defined the triangulated hull of the orbit category. In a similar way we show that $\mathcal{E} / F$ embeds into a certain ambient exact category and give sufficient conditions to ensure that $\mathcal{E} / F$ is an extension-closed subcategory of the ambient category. To be more precise, let $\mathcal{P}$ be the full subcategory of $\mathcal{E}$ determined by its projective objects and $\operatorname{gpr}(\mathcal{P} / F)$ be the category of finitely-generated Gorenstein-projective modules over $\mathcal{P} / F$ (see Definition 25). The category $\operatorname{gpr}(\mathcal{P} / F)$ is an exact category, it is even a Frobenius category. Inspired by a result of Chen [8], we prove that there is a full and faithful functor $\mathcal{E} / F \hookrightarrow \operatorname{gpr}(\mathcal{P} / F)$. We prove the following theorem which is Theorem 43 of this note.

Theorem. Suppose that $\underline{\mathcal{E}} / \underline{F}$ is equivalent to its triangulated hull. If $\mathcal{E} / F$ has split idempotents, then $\mathcal{E} / F$ is closed under extensions in $\operatorname{gpr}(\mathcal{P} / F)$. Moreover, the induced exact structure on $\mathcal{E} / F$ makes the canonical projection $\mathcal{E} \rightarrow \mathcal{E} / F$ exact and makes $\mathcal{E} / F$ a Frobenius category whose stable category is triangle equivalent to $\underline{\mathcal{E}} / \underline{F}$.

The ambient Frobenius category $\operatorname{gpr}(\mathcal{P} / F)$ should not be considered as the exact (or Frobenius) hull of $\mathcal{E} / F$ since it may be too large. Still, $\operatorname{gpr}(\mathcal{P} / F)$ can be considered as a canonical ambient category for $\mathcal{E} / F$ since the functor $\mathcal{E} / F \hookrightarrow \operatorname{gpr}(\mathcal{P} / F)$ is induced (in a sense made precise in Definition 27) by the Yoneda functor $\mathcal{E} / F \hookrightarrow \operatorname{Mod}(\mathcal{E} / F)$. Even more, the triangulated structure on the stable category $\operatorname{gpr}(\mathcal{P} / F)$ and on the triangulated hull of $\underline{\mathcal{E}} / \underline{F}$ are compatible in the following sense: the inclusion $\mathcal{E} / F \hookrightarrow \operatorname{gpr}(\mathcal{P} / F)$ induces a fully faithful triangulated functor from the triangulated hull of $\underline{\mathcal{E}} / \underline{F}$ into $\underline{\operatorname{gpr}}(\mathcal{P} / F)$ (see Theorem 41).

Orbit categories have appeared (perhaps sometimes in an implicit way) in the work of many mathematicians (see for instances [15] [6], [21], [13], [28] and [32]). One of our main motivations for studying orbit categories of Frobenius categories comes from representation theory, and more concretely, from the additive categorification of acyclic cluster algebras introduced in [6] (see also [11] and [23]). In particular, we are interested in the case where $\mathcal{E}$ is in addition a Krull-Schmidt category. In general $\mathcal{E} / F$ fails to be Krull-Schmidt. Yet, under certain finiteness conditions on $F$ (stated explicitly in Section 6) we are able to give sufficient conditions to prove the following theorem which is Theorem 42 of this note.

Theorem. If $\mathcal{E}$ is Krull-Schmidt (and $F$ is as discribed above) then the completed orbit category $\mathcal{E} \widehat{/ F}$ admits the structure of a Frobenius category which makes the canonical functor $\mathcal{E} \rightarrow \mathcal{E} \widehat{/ F}$ exact and whose stable category is triangle equivalent to $\underline{\mathcal{E}} / \underline{F}$.

The completed orbit category is defined just as the usual orbit category by replacing the direct sum in (1.1) by the direct product. The composition formula (2.2) of usual orbit categories defines a composition in the completed orbit category provided that, for every pair of objects $X$ and $Y$, the group $\mathcal{E}\left(X, F^{l}(Y)\right)$ vanishes for $l \ll 0$. This last theorem will allow us to give an explicit categorification of families of finite-type cluster algebras with coefficients. In particular, we obtain a categorification of all skew-symmetric finitetype cluster algebras with universal coefficients. We would like to stress that completed 
orbit categories already appeared in [17] where they are used to define continuous cluster categories.

This article is organized as follows. In the Section 2 we survey Keller's construction of the triangulated hull associated to the orbit category of a triangulated category. In section 3 we recall a Theorem of Chen which shows that any Frobenius category is equivalent to an extension-closed exact subcategory of the Frobenius category formed by Gorensteinprojective modules over some additive category. This theorem will allow us to define the embedding of $\mathcal{E} / F$ into $\operatorname{gpr}(\mathcal{P} / F)$. In section 4 we prove some general results on usual and completed orbit categories which will be used intensively. In section 5 we prove the compatibility of the triangulated structure of $\operatorname{gpr}(\mathcal{P} / F)$ and of the triangulated hull of $\underline{\mathcal{E}} / \underline{F}$. In section 6 we give a proof of the theorems stated above. In section 7 we apply our results on completed orbit categories to the context of Nakajima categories associated to Dynkin quivers to introduce explicit categorifications of families of finite-type cluster algebras with coefficients. In particular, we obtain a categorification of all skew-symmetric finite-type cluster algebras with universal coefficients.

Acknowledgments. The work in this article is exposed in my Ph.D. thesis, supervised by Professors Bernhard Keller and Lauren Williams. I would like to thank them for their guidance and patience. I am grateful to Raika Dehy, Patrick Le Meur, Yann Palu and Pierre-Guy Plamondon for the useful discussions. The final version of this note was written at the Max Planck Institut for Mathematics, Bonn, during the 2015 fall semester. The author is deeply grateful to this institution for the financial support and for providing ideal working conditions.

\section{REMinders ON DG CATEGORIES AND THEIR ORBIT CATEGORIES}

Throughout this chapter, we will freely use the theory of exact categories first introduced by Quillen in [29]. Our main reference for this theory is the refined treatment presented in [18, Appendix A] and the systematic study of [7]. We will also use the basic facts about Frobenius categories. The reader is referred to [16] for a treatment of this topic. The set of morphisms between two objects $X$ and $Y$ of a category $\mathcal{A}$ is denoted by $\mathcal{A}(X, Y)$. If $k$ is a ring and $\mathcal{A}$ an additive $k$-category, a right $\mathcal{A}$-module is by definition a $k$-linear functor $M: \mathcal{A}^{\text {op }} \rightarrow \operatorname{Mod} k$, where $\operatorname{Mod} k$ is the category of all right $k$-modules. The morphism space between two $\mathcal{A}$-modules $L$ and $M$ is denoted by $\operatorname{Hom}_{\mathcal{A}}(L, M)$ or simply $\operatorname{Hom}(L, M)$ when there is no risk of confusion.

2.1. Pretriangulated $\mathbf{d g}$ categories. In this section we recall some facts about dg categories and introduce notation. Our main reference for these results are [20] and [10]. We work over an arbitrary field $k$. In this section, all categories, dg categories, functors, $\mathrm{dg}$ functors, etc. are assumed to be $k$-linear.

A dg category $\mathcal{B}$ is a category whose morphism spaces have the structure of a differential graded $k$-module, or equivalently, a complex of $k$-modules. For a dg category $\mathcal{B}$ we denote by $Z^{0}(\mathcal{B})$ the category with the same objects as $\mathcal{B}$, and with morphisms $Z^{0}(\mathcal{B})(X, Y)=$ $Z^{0}(\mathcal{B}(X, Y))$. The category $H^{0}(\mathcal{B})$ is defined analogously. Let $\mathcal{C}_{d g}(k)$ be the $\operatorname{dg}$ category of differential graded $k$-modules.

Notation 1. Let $\mathcal{B}$ be a dg category. A right $d g \mathcal{B}$-module is a dg functor $L: \mathcal{B}^{o p} \rightarrow \mathcal{C}_{d g}(k)$. We denote by $\mathcal{C}_{d g}(\mathcal{B})$ the $\mathrm{dg}$ category of right dg $\mathcal{B}$-modules. Denote by $\mathcal{C}(\mathcal{B})$ and $\mathcal{H}(\mathcal{B})$ the categories $Z^{0}\left(\mathcal{C}_{d g}(\mathcal{B})\right)$ and $H^{0}\left(\mathcal{C}_{d g}(\mathcal{B})\right)$, respectively. The derived category $\mathcal{D}(\mathcal{B})$ is the localization of $\mathcal{C}(\mathcal{B})$ with respect to the quasi-isomorphisms. 
Remark 2. The category $\mathcal{C}(\mathcal{B})$ admits an exact structure by defining an admissible short exact sequence (or conflation) to be a sequence $L \rightarrow M \rightarrow N$ such that the underlying sequence of graded $\mathcal{B}$-modules is split short exact. Endowed with this structure, $\mathcal{C}(\mathcal{B})$ becomes a Frobenius category whose stable category is $\mathcal{H}(\mathcal{B})$ (cf. [19, Lemma 2.2]).

Example 3. Let $\mathcal{A}$ be an additive category. We consider $\mathcal{A}$ as a dg category whose morphism complexes are concentrated in degree 0 . Then, the objects of $\mathcal{C}_{d g}(\mathcal{A})$ can be thought of as complexes of right $\mathcal{A}$-modules. The morphism complex

$$
\mathcal{H o m}(X, Y):=\mathcal{C}_{d g}(\mathcal{A})(X, Y)
$$

between the complexes $X=\cdots \rightarrow X_{i} \rightarrow X_{i+1} \rightarrow \cdots$ and $Y=\cdots \rightarrow Y_{i} \rightarrow Y_{i+1} \rightarrow \cdots$ has as $n^{\text {th }}$ component the $k$-module

$$
\mathcal{H o m}^{n}(X, Y)=\prod_{i \in \mathbb{Z}} \operatorname{Hom}_{\mathcal{A}}\left(X_{i}, Y_{i+n}\right) .
$$

The differential on $\mathcal{H o m}(X, Y)$ is given by

$$
d(f)=\left(f_{i} d_{Y}-(-1)^{n} d_{X} f_{i+1}\right)_{i \in \mathbb{Z}}
$$

for $f=\left(f_{i}\right)_{i \in \mathbb{Z}} \in \mathcal{H o m}^{n}(X, Y)$. It follows that $\mathcal{C}(\mathcal{A})$ is the category of chain complexes of $\mathcal{A}$-modules and that $\mathcal{H}(\mathcal{A})$ is the homotopy category of chain complexes of $\mathcal{A}$-modules.

Notation 4 . If $\mathcal{A}$ is an additive category we denote by $C(\mathcal{A})_{d g}$ the dg category of complexes with components in $\mathcal{A}$. Its objects are the complexes with components in $\mathcal{A}$ and the morphism complex between two objects is defined in the same way as in Example 3.

Warning 5. Notice the difference between the categories $\mathcal{C}_{d g}(\mathcal{A})$ and $C(\mathcal{A})_{d g}$.

Remark 6. For each dg category $\mathcal{B}$, the Yoneda functor $\mathcal{B} \rightarrow \mathcal{C}_{d g}(\mathcal{B})$ takes an object $X$ to the representable dg module $\mathcal{C}_{d g}(\mathcal{B})(-, X)$. Slightly abusing, we also call the induced functors $Z^{0}(\mathcal{B}) \rightarrow \mathcal{C}(\mathcal{B})$ and $H^{0}(\mathcal{B}) \rightarrow \mathcal{H}(\mathcal{B})$ Yoneda functors.

Definition 7. We call $\mathcal{B}$ pretriangulated, if the image of the Yoneda functor $Z^{0}(\mathcal{B}) \hookrightarrow$ $\mathcal{C}(\mathcal{B})$ is closed under translations and extensions (with respect to the exact structure of Remark 2). If $\mathcal{B}$ is pretriangulated then the category $Z^{0}(\mathcal{B})$ is a Frobenius subcategory of $\mathcal{C}(\mathcal{B})$. A $d g$ enhancement of a triangulated category $\mathcal{T}$ is a pair $(\mathcal{B}, \epsilon)$, where $\mathcal{B}$ is a pretriangulated $\mathrm{dg}$ category and $\epsilon: H^{0}(\mathcal{B}) \rightarrow \mathcal{T}$ is a triangle equivalence. We call $\mathcal{T}$ algebraic if it admits a dg enhancement.

Example 8. Let $\mathcal{A}$ be an additive category. The $\operatorname{dg}$ category $C(\mathcal{A})_{d g}$ is a typical example of a pretriangulated $\mathrm{dg}$ category. If $\mathcal{B}$ is a pretriangulated $\mathrm{dg}$ category and $\mathcal{B}^{\prime}$ is a subcategory of $\mathcal{B}$ such that $Z^{0}\left(\mathcal{B}^{\prime}\right)$ is closed in $Z^{0}(\mathcal{B})$ under shifts and extensions, then $\mathcal{B}^{\prime}$ is pretriangulated. In particular, if $\mathcal{A}$ is exact, $\mathcal{P}$ is a subcategory of $\mathcal{A}$ closed under direct sums and we let $\mathcal{A c}(\mathcal{P})_{d g}$ be the full subcategory of $C(\mathcal{A})_{d g}$ formed by the acyclic complexes with components in $\mathcal{P}$, then $\mathcal{A c}(\mathcal{P})_{d g}$ is a pretriangulated dg category.

Example 9. Let $\mathcal{E}$ be a Frobenius category and let $\mathcal{P}$ be its full subcategory of projectiveinjective objects. We denote by $\underline{\mathcal{E}}$ the stable category of $\mathcal{E}$, i.e. the quotient category of $\mathcal{E}$ by the ideal of morphisms factorizing through elements of $\mathcal{P}$. By Example 8, the $\mathrm{dg}$ category $\mathcal{A} c(\mathcal{P})_{d g}$ is pretriangulated. Moreover $\mathcal{A} c(\mathcal{P})_{d g}$ is a (canonical) dg enhancement of $\mathcal{E}$. Indeed: the category $Z^{0}\left(\mathcal{A} c(\mathcal{P})_{d g}\right)$ identifies with the category of acyclic complexes with components in $\mathcal{P}$. Is easy to see that there is triangle equivalence

$$
Z^{0}: H^{0}\left(\mathcal{A c}(\mathcal{P})_{d g}\right) \stackrel{\sim}{\longrightarrow} \underline{\mathcal{E}}
$$

which takes a complex $P^{\cdot}$ to its 0 -cycles $Z^{0}\left(P^{\cdot}\right)$ cf. [25, Section 1.5]. 
Definition 10. If $\mathcal{B}$ is an arbitrary small $\mathrm{dg}$ category and $\mathcal{B}^{\prime}$ is a $\mathrm{dg}$ category, then the category of $\operatorname{dg}$ functors $\mathcal{H o m}\left(\mathcal{B}, \mathcal{B}^{\prime}\right)$ is a $\operatorname{dg}$ category in a natural way (see [20, Section 2.3]). There is a universal $\mathrm{dg}$ functor $\mathcal{B} \rightarrow \operatorname{pretr}(\mathcal{B})$ to a pretriangulated dg category $\operatorname{pretr}(\mathcal{B})$, i.e. a functor inducing an equivalence of $\operatorname{dg}$ categories $\mathcal{H o m}\left(\mathcal{B}, \mathcal{B}^{\prime}\right) \rightarrow \mathcal{H o m}\left(\operatorname{pretr}(\mathcal{B}), \mathcal{B}^{\prime}\right)$ for each pretriangulated $d g$ category $\mathcal{B}^{\prime}$. The $\operatorname{dg}$ category $\operatorname{pretr}(\mathcal{B})$ is the pretriangulated hull of $\mathcal{B}$.

Remark 11. The pretriangulated hull of $\mathcal{B}$ is constructed explicitly in [4] (where it is denoted by Pre- $\left.\operatorname{tr}^{+}(\mathcal{B})\right)$, cf. also [10] and [33].

2.2. Quasi-functors. Let $\mathcal{B}$ and $\mathcal{B}^{\prime}$ be two dg categories. A $\mathcal{B}$ - $\mathcal{B}^{\prime}$-bimodule $M$ is an object of $\mathcal{C}_{d g}\left(\mathcal{B}^{\text {op }} \otimes \mathcal{B}^{\prime}\right)$, i.e. $M$ is a left $\mathcal{B}$-module and a right $\mathcal{B}^{\prime}$-module. Let $\operatorname{rep}\left(\mathcal{B}, \mathcal{B}^{\prime}\right)$ be the full subcategory of the derived category $\mathcal{D}\left(\mathcal{B}^{\text {op }} \otimes \mathcal{B}^{\prime}\right)$ formed by the bimodules $X$ such that the derived tensor product

$$
? \stackrel{L}{\otimes_{\mathcal{B}}} X: \mathcal{D}(\mathcal{B}) \rightarrow \mathcal{D}\left(\mathcal{B}^{\prime}\right)
$$

takes the representable $\mathcal{B}$-modules to objects which are isomorphic to a representable $\mathcal{B}^{\prime}$ modules.

Remark 12. Every object $X$ in $\mathcal{D}\left(\mathcal{B}^{\text {op }} \otimes \mathcal{B}^{\prime}\right)$ is isomorphic to a cofibrant object of $\mathcal{D}\left(\mathcal{B}^{\text {op }} \otimes\right.$ $\left.\mathcal{B}^{\prime}\right)$. Therefore, in practice we will always assume that every object in $\operatorname{rep}\left(\mathcal{B}, \mathcal{B}^{\prime}\right)$ is cofibrant and we will consider it as a bimodule in $\mathcal{C}_{d g}\left(\mathcal{B}^{\text {op }} \otimes \mathcal{B}^{\prime}\right)$. In particular, we require that $X(B$, ?) is quasi-isomorphic to a representable $\mathcal{B}^{\prime}$-module for each object $B$ of $\mathcal{B}$. The category of $\mathcal{B}^{\prime}-$ modules which are quasi-isomorphic to a representable dg module is equivalent to $H^{0}\left(\mathcal{B}^{\prime}\right)$. Therefore an object of $\operatorname{rep}\left(\mathcal{B}, \mathcal{B}^{\prime}\right)$ defines a functor

$$
H^{0}(\mathcal{B}) \rightarrow H^{0}\left(\mathcal{B}^{\prime}\right)
$$

For this reason, the objects in $\operatorname{rep}\left(\mathcal{B}, \mathcal{B}^{\prime}\right)$ are called quasi-functors.

The bimodule bicategory rep has as objects all small dg categories; the morphism category between two objects $\mathcal{B}$ and $\mathcal{B}^{\prime}$ is $\operatorname{rep}\left(\mathcal{B}, \mathcal{B}^{\prime}\right)$; the composition bifunctor

$$
\operatorname{rep}\left(\mathcal{B}^{\prime}, \mathcal{B}^{\prime \prime}\right) \times \operatorname{rep}\left(\mathcal{B}, \mathcal{B}^{\prime}\right) \rightarrow \operatorname{rep}\left(\mathcal{B}, \mathcal{B}^{\prime \prime}\right)
$$

is given by the derived tensor product $(X, Y) \mapsto X \stackrel{L}{\otimes_{\mathcal{B}^{\prime}}} Y$. For each dg functor $F: \mathcal{B} \rightarrow$ $\mathcal{B}^{\prime}$, we have the dg bimodule

$$
M_{F}:\left(B, B^{\prime}\right) \mapsto \mathcal{B}^{\prime}\left(B^{\prime}, F(B)\right)
$$

which clearly belongs to $\operatorname{rep}\left(\mathcal{B}, \mathcal{B}^{\prime}\right)$.

2.3. Orbit categories. Let $\mathcal{A}$ be a $k$-linear category and $F: \mathcal{A} \rightarrow \mathcal{A}$ be an automorphism. By definition, the orbit category $\mathcal{A} / F$ has the same objects as $\mathcal{A}$ and the morphisms between two objects $X$ and $Y$ are given by

$$
\mathcal{A} / F(X, Y)=\bigoplus_{l \in \mathbb{Z}} \mathcal{A}\left(X, F^{l}(Y)\right)
$$

The composition is given by the formula

$$
\left(f_{a}\right) \circ\left(g_{b}\right)=\left(\sum_{a+b=c} F^{b}\left(f_{a}\right) \circ g_{b}\right),
$$

where $f_{a}: Y \rightarrow F^{a}(Z), g_{b}: X \rightarrow F^{b}(Y)$ and $a, b \in \mathbb{Z}$. Let $p: \mathcal{A} \rightarrow \mathcal{A} / F$ be the canonical projection functor. It is endowed with a canonical isomorphism of functors $\phi: p \rightarrow p \circ F$ given by $\phi_{X}=\left(\ldots, 0,1_{F^{-1}(X)}, 0, \ldots\right)$ for each object $X$ of $\mathcal{A}$. Let $\mathcal{A}^{\prime}$ be another $k$-linear 
category. An $F$-invariant functor from $\mathcal{A}$ to $\mathcal{A}^{\prime}$ is given by a pair $(G, \phi)$, where $G: \mathcal{A} \rightarrow \mathcal{A}^{\prime}$ is a $k$-linear functor and $\phi: G \rightarrow G \circ F$ is an isomorphism of functors. A morphism of $F$-invariant functors $(G, \phi) \rightarrow\left(G^{\prime}, \phi^{\prime}\right)$ is given by a morphism of functors $\alpha: G \rightarrow G^{\prime}$ such that the square

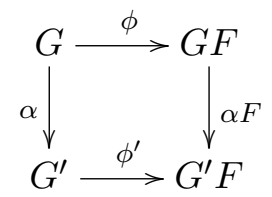

commutes. In this way, we obtain the category $\operatorname{inv}_{F}\left(\mathcal{A}, \mathcal{A}^{\prime}\right)$ of $F$-invariant functors. In particular, $(p, \phi)$ is an $F$-invariant functor. The orbit category satisfies the following universal property.

Theorem 13. ([1, Corollary 3.5]. See also [22]) Let fun ${ }_{k}\left(\mathcal{A} / F, \mathcal{A}^{\prime}\right)$ be the category of $k$ linear functors from $\mathcal{A} / F$ to $\mathcal{A}^{\prime}$. The functor

$$
\operatorname{fun}_{k}\left(\mathcal{A} / F, \mathcal{A}^{\prime}\right) \rightarrow \operatorname{inv}_{F}\left(\mathcal{A}, \mathcal{A}^{\prime}\right), G \mapsto G \circ p
$$

is an isomorphism of categories.

Remark 14. We may suppose without any risk that $F$ is an autoequivalence. The theoretical justification for this can be found in Section 7 of [2].

The $d g$ orbit category associated to a $d g$ category $\mathcal{B}$ and a quasi-functor in $\operatorname{rep}(\mathcal{B}, \mathcal{B})$ is defined by a universal property. This property can be thought of as a lift of Theorem 13 to the dg world (see Remark 19 below). To state properly the universal property of the dg orbit categories we need to introduce the following definition.

Definition 15. Suppose that $\mathcal{B}$ is small and that $F \in \operatorname{rep}(\mathcal{B}, \mathcal{B})$ is given by a cofibrant bimodule. For a dg category $\mathcal{B}^{\prime}$, define $\widetilde{\operatorname{eff}}\left(\mathcal{B}, F, \mathcal{B}^{\prime}\right)$ to be the category whose objects are given by pairs $(P, \phi)$ where

- $P$ is a quasi-functor in $\operatorname{rep}\left(\mathcal{B}, \mathcal{B}^{\prime}\right)$,

- $\phi: P \rightarrow P F$ is a quasi-isomorphism of dg bimodules,

- the morphisms from $(P, \phi)$ to $\left(P^{\prime}, \phi^{\prime}\right)$ are obtained the morphisms $f: P \rightarrow P^{\prime}$ of dg bimodules, such that $\phi^{\prime} \circ f=(f F) \circ \phi$ in the category of dg bimodules. In other words, the following diagram commutes

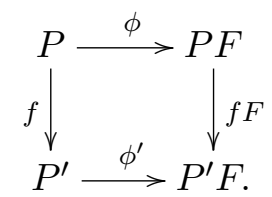

Let eff $\left(\mathcal{B}, F, \mathcal{B}^{\prime}\right)$ be the localization of $\widetilde{\operatorname{eff}}\left(\mathcal{B}, F, \mathcal{B}^{\prime}\right)$ with respect to the morphisms $f$ which are quasi-isomorphisms of dg bimodules.

Remark 16. The name eff comes from the french word effaçable which means erasable.

Theorem 17. ([21, Theorem 3 (b) $])$ Let $\mathcal{B}$ a dg category and $F \in \operatorname{rep}(\mathcal{B}, \mathcal{B})$. Then the 2 functor $\operatorname{eff}(\mathcal{B}, F, ?)$ is 2-representable, i.e. there exist a $d g$ category $\mathcal{B} / F$ and a pair $\left(P_{0}, \phi_{0}\right)$ in $\operatorname{eff}(\mathcal{B}, F, \mathcal{B} / F)$ such that for every small $d g$ category $\mathcal{B}^{\prime}$, the functor

$$
\operatorname{rep}\left(\mathcal{B} / F, \mathcal{B}^{\prime}\right) \rightarrow \operatorname{eff}\left(\mathcal{B}, F, \mathcal{B}^{\prime}\right), G \mapsto G \circ P_{0}
$$

is an equivalence. 
We call $\mathcal{B} / F$ the $d g$ orbit category associated to $\mathcal{B}$ and $F$. If $\mathcal{B}$ is a dg category endowed with an endomorphism $F: \mathcal{B} \rightarrow \mathcal{B}$ inducing an equivalence $H^{0}(F): H^{0}(\mathcal{B}) \rightarrow H^{0}(\mathcal{B})$, then $\mathcal{B} / F:=\mathcal{B} / M_{F}$ can be described explicitly as follows: The objects of $\mathcal{B} / F$ are the same as the objects of $\mathcal{B}$. For $X, Y \in \mathcal{B} / F$, we have

$$
\mathcal{B} / F(X, Y):=\operatorname{colim}_{p} \bigoplus_{n \geq 0} \mathcal{B}\left(F^{n}(X), F^{p}(Y)\right),
$$

where the transitions maps are given by $F$

$$
\bigoplus_{n \geq 0} \mathcal{B}\left(F^{n}(X), F^{p}(Y)\right) \stackrel{F}{\longrightarrow} \bigoplus_{n \geq 0} \mathcal{B}\left(F^{n}(X), F^{p+1}(Y)\right) .
$$

Combining Theorem 17 with the universal property of the pretriangulated hull we obtain the following universal property:

Theorem 18. ([21, Theorem 4]) Let $\mathcal{B}$ be a pretriangulated $d g$ category and $F \in \operatorname{rep}(\mathcal{B}, \mathcal{B})$. Then for any pretriangulated $d g$ category $\mathcal{B}^{\prime}$ there is an equivalence of categories

$$
\operatorname{rep}\left(\operatorname{pretr}(\mathcal{B} / F), \mathcal{B}^{\prime}\right) \stackrel{\sim}{\longrightarrow} \operatorname{eff}\left(\mathcal{B}, F, \mathcal{B}^{\prime}\right) .
$$

Remark 19. If $(P, \phi) \in \operatorname{eff}\left(\mathcal{B}, F, \mathcal{B}^{\prime}\right)$ then $H^{0}(P)$ is an $H^{0}(F)$-invariant functor. By Theorem $13 H^{0}(P)$ induces a functor $G: H^{0}(\mathcal{B}) / H^{0}(F) \rightarrow H^{0}\left(\mathcal{B}^{\prime}\right)$. By Theorem $17(P, \phi)$ corresponds to a quasi-functor $\tilde{G}$ such that $H^{0}(\tilde{G})=G$.

The $\mathrm{dg}$ orbit category is functorial in $(\mathcal{B}, F)$ in the following sense.

Lemma 20. (cf. [21, Section 9.4]) Let

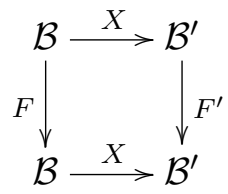

be an square in rep and let $\gamma: F^{\prime} X \rightarrow X F$ be an isomorphism in $\operatorname{rep}\left(\mathcal{B}, \mathcal{B}^{\prime}\right)$. Then there is a morphism $\bar{X}: \mathcal{B} / F \rightarrow \mathcal{B}^{\prime} / F^{\prime}$ such that if

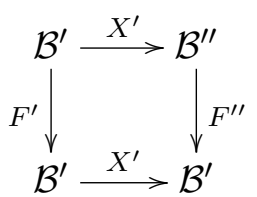

is another diagram in rep as above, then the quasi-functors $\overline{X^{\prime}} \circ \bar{X}$ and $\overline{X^{\prime} \circ X}$ are isomorphic.

Definition 21. Let $\mathcal{T}=H^{0}(\mathcal{B})$ be an algebraic triangulated category, and $\widetilde{F}: \mathcal{B} \rightarrow \mathcal{B}$ a dg functor inducing an equivalence $F: \mathcal{T} \rightarrow \mathcal{T}$. Then the triangulated hull of $\mathcal{T} / F$ is defined as the triangulated category

$$
H^{0}(\operatorname{pretr}(\mathcal{B} / \widetilde{F})) .
$$

Definition 22. Let $\mathcal{T}$ be a triangulated category endowed with an autoequivalence $F$ : $\mathcal{T} \rightarrow \mathcal{T}$. Suppose that $\mathcal{T}_{d g}$ is a dg enhancement of $\mathcal{T}$ and that $\widetilde{F} \in \operatorname{rep}\left(\mathcal{T}_{d g}, \mathcal{T}_{d g}\right)$ is a dg lift of $F$. The triangulated hull of $(\mathcal{T}, F)$ with respect to $\mathcal{T}_{d g}$ is the triangulated category $H^{0}\left(\operatorname{pretr}\left(\mathcal{T}_{d g} / \widetilde{F}\right)\right)$. We say that $\mathcal{T} / F$ is triangulated with respect to $\left(\mathcal{T}_{d g}, \tilde{F}\right)$ if $\mathcal{T} / F$ is equivalent to the triangulated hull defined by $\mathcal{T}_{d g}$ and $\widetilde{F}$. 
Remark 23. Let $\mathcal{H}$ be a hereditary abelian category and $F: \mathcal{D}^{b}(\mathcal{H}) \rightarrow \mathcal{D}^{b}(\mathcal{H})$ an autoequivalence. In [21], Keller gives sufficient conditions on $F$ to ensure that $\mathcal{D}^{b}(\mathcal{H}) / F$ is triangulated with respect to $\mathcal{D}^{b}(\mathcal{H})_{d g}$.

\section{The ambient Frobenius Category}

3.1. Frobenius categories and Gorenstein-projective modules. In this subsection we recall a general result on Frobenius categories due to Chen [8]. It allows us to embed any Frobenius category $\mathcal{E}$ into a module category (over an additive category). More precisely, let $\mathcal{P}$ be the full subcategory of $\mathcal{E}$ formed by its projective objects, then $\mathcal{E}$ is equivalent, as an exact category, to an extension-closed exact subcategory of $\operatorname{gpr}(\mathcal{P})$, the category of finitely-generated Gorenstein-projective (or maximal Cohen-Macaulay) modules over $\mathcal{P}$. This theorem is crucial for our construction of an ambient Frobenius category in which the orbit category $\mathcal{E} / F$ embeds.

Notation 24. Let $\mathcal{A}$ be an additive $\mathbb{Z}$-category. We denote by $\operatorname{Mod}(\mathcal{A})$ the category of all right modules over $\mathcal{A}$ and by $\bmod (\mathcal{A})$ its full subcategory of finitely presented modules. We let $\operatorname{proj}(\mathcal{A})$ be the full subcategory of $\bmod (\mathcal{A})$ formed by the finitely-generated projective $\mathcal{A}$-modules.

Definition 25. An $\mathcal{A}$-module $M$ is finitely generated Gorenstein projective if there is an acyclic complex

$$
P_{M}: \cdots \rightarrow P_{1} \rightarrow P_{0} \rightarrow P^{0} \rightarrow P^{1} \rightarrow \cdots
$$

of objects in $\operatorname{proj}(\mathcal{A})$ such that $M \cong \operatorname{cok}\left(P_{1} \rightarrow P_{0}\right)$ and the complex $\operatorname{Hom}_{\mathcal{A}}\left(P_{M}, P^{\prime}\right)$ is still acyclic for each module $P^{\prime}$ in $\operatorname{proj}(\mathcal{A})$. Denote by $\operatorname{gpr}(\mathcal{A})$ the full subcategory of $\bmod (\mathcal{A})$ formed by the Gorenstein projective modules. In the situation described above we call $P_{M}$ a complete projective resolution of $M$.

Notice that every finitely generated projective $\mathcal{A}$-module $P$ lies in $\operatorname{gpr}(\mathcal{A})$, since we may take its complete resolution as $\cdots \rightarrow 0 \rightarrow P \stackrel{\sim}{\longrightarrow} P \rightarrow 0 \rightarrow \cdots$.

Lemma 26. The category $\operatorname{gpr}(\mathcal{A})$ is a Frobenius category whose subcategory of projectiveinjective objects is $\operatorname{proj}(\mathcal{A})$, the category of finitely-generated projective $\mathcal{A}$-modules.

Proof. By [3, Proposition 5.1] the category $\operatorname{gpr}(\mathcal{A})$ is an extension-closed subcategory of $\operatorname{Mod}(\mathcal{A})$ and thus, it is an exact category. Let $P$ and $P^{\prime}$ be finitely-generated projective $\mathcal{A}$-modules. A complex of the form $(\cdots \rightarrow 0 \rightarrow P=P \rightarrow 0 \rightarrow \cdots)$ is acyclic and remains acyclic after applying the functor $\operatorname{Hom}\left(?, P^{\prime}\right)$. Therefore $\operatorname{proj}(\mathcal{A})$ identifies with the subcategory of projective objects of $\operatorname{gpr}(\mathcal{A})$. Let $M$ be a module in $\operatorname{gpr}(\mathcal{A})$ and $P_{M}=(\cdots \rightarrow$ $\left.P_{1} \rightarrow P_{0} \rightarrow P^{0} \rightarrow P^{1} \rightarrow \cdots\right)$ a complete resolution of $M$. Since the complex $\operatorname{Hom}\left(P_{M}, P\right)$ is acyclic, we have that $\operatorname{Ext}_{\mathcal{A}}^{1}(M, P)=0$. Therefore $P$ is also injective $\operatorname{in} \operatorname{gpr}(\mathcal{A})$. The sequences $0 \rightarrow Z^{-1}\left(P_{M}\right) \rightarrow P_{0} \rightarrow M \rightarrow 0$ and $0 \rightarrow M \rightarrow P^{0} \rightarrow Z^{1}\left(P_{M}\right) \rightarrow 0$ are short exact sequences of $\bmod (\mathcal{P})$ which lie in $\operatorname{gpr}(\mathcal{P})$. Therefore $\operatorname{gpr}(\mathcal{A})$ has enough projectives and enough injectives. Moreover each injective object in $\operatorname{gpr}(\mathcal{A})$ must be projective too. This completes the proof.

Definition 27. Let $\mathcal{V}$ be a subcategory of $\mathcal{A}$. Then the assignment

$$
\left.X \mapsto \mathcal{A}(?, X)\right|_{\mathcal{V}}
$$

induces a functor $\mathcal{A} \rightarrow \operatorname{Mod}(\mathcal{V})$, which we call the restricted Yoneda functor.

Lemma 28. Let $\mathcal{E}$ be an exact category and let $\mathcal{P}$ be its full subcategory of projective objects. Let $0 \rightarrow A \rightarrow B \rightarrow C \rightarrow 0$ be an exact sequence in $\mathcal{E}$. Then the induced sequence

$$
\left.\left.\left.0 \longrightarrow \mathcal{E}(?, A)\right|_{\mathcal{P}} \longrightarrow \mathcal{E}(?, B)\right|_{\mathcal{P}} \longrightarrow \mathcal{E}(?, C)\right|_{\mathcal{P}} \longrightarrow 0
$$


is exact in $\operatorname{Mod}(\mathcal{P})$.

Proof. This follows from the fact that $\mathcal{E}(P, ?)$ is exact for every $P \in \mathcal{P}$.

Corollary 29. Suppose $\mathcal{E}$ is a Frobenius category. Then the essential image of the restricted Yoneda functor $\mathcal{E} \rightarrow \operatorname{Mod}(\mathcal{P})$ is contained in $\operatorname{gpr}(\mathcal{P})$.

Proof. It follows from Lemma 28 that the complete resolution

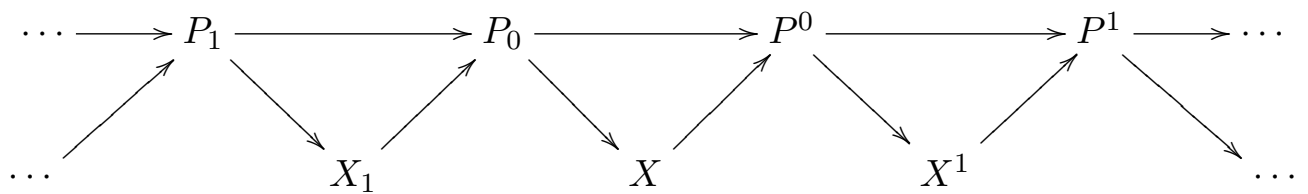

obtained by splicing the admissible short exact sequences $0 \rightarrow X_{i+1} \rightarrow P_{i} \rightarrow X_{i} \rightarrow 0$ and $0 \rightarrow X^{i} \rightarrow P^{i} \rightarrow X^{i+1} \rightarrow 0$ for $i \geq 0$ and $X_{0}=X=X^{0}$ is sent to the complete resolution

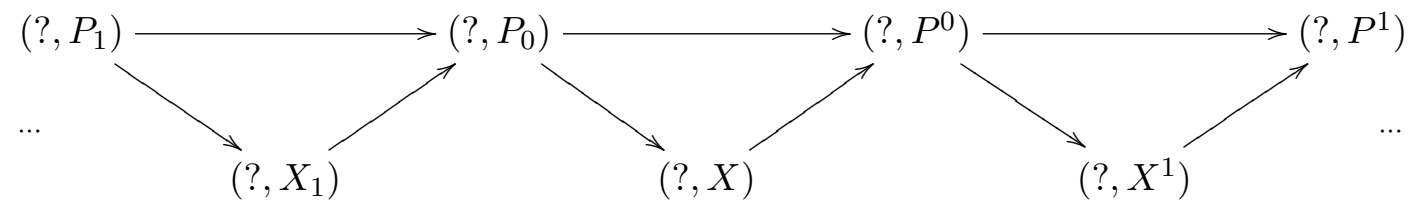

where we abbreviate $\left.\mathcal{E}(?, ?)\right|_{\mathcal{P}}$ by $(?, ?)$. The claim follows easily.

The following theorem is a version of [8, Theorem 4.2] that can be deduced from Lemma 28 and Corollary 29. It allows us to think of $\mathcal{E}$ as a full exact subcategory of $\operatorname{gpr}(\mathcal{P})$.

Theorem 30. The restricted Yoneda functor $\mathcal{E} \rightarrow \operatorname{gpr}(\mathcal{P})$ is full and faithful. Moreover, its essential image is an exact-closed subcategory of $\operatorname{gpr}(\mathcal{P})$.

3.2. The ambient Frobenius category for the orbit category. Suppose that $\mathcal{E}$ is a essentially small Frobenius category endowed with an exact automorphism $F: \mathcal{E} \rightarrow \mathcal{E}$. Let $p: \mathcal{E} \rightarrow \mathcal{E} / F$ be the natural projection. It induces a pair of adjoint functors

$$
\begin{gathered}
\operatorname{Mod}(\mathcal{E}) \\
\pi|\uparrow| p^{*} \\
\operatorname{Mod}(\mathcal{E} / F),
\end{gathered}
$$

where $p^{*}$ is the restriction functor and its left adjoint $\pi$ is the extension of $p$ to $\operatorname{Mod}(\mathcal{A})$ (cf. [26, Lemma 2.4]). It is clear that the full subcategory of $\mathcal{E} / F$ defined by the objects in $\mathcal{P}$ is equivalent to $\mathcal{P} / F$. Therefore, we will consider $\mathcal{P} / F$ as a full subcategory of $\mathcal{E} / F$.

Theorem 31. The restricted Yoneda functor $\mathcal{E} / F \rightarrow \operatorname{Mod}(\mathcal{P} / F)$ is full and faithful, its essential image is contained in $\operatorname{gpr}(\mathcal{P} / F)$ and there is a commutative diagram

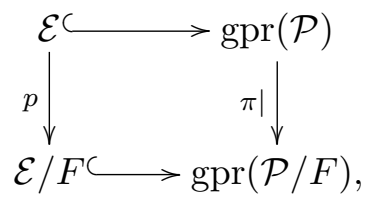

where $\pi \mid$ denotes is the restriction of $\pi$ to $\operatorname{gpr}(\mathcal{P})$.

Recall that $\mathcal{A} c(\mathcal{P})_{d g}$ is the canonical dg enhancement of $\underline{\mathcal{E}}$ (see Example 9). Let $\widetilde{F}$ : $\mathcal{A} c(\mathcal{P})_{d g} \rightarrow \mathcal{A} c(\mathcal{P})_{d g}$ be the dg functor given by $F$ componentwise and let $\underline{F}: \underline{\mathcal{E}} \rightarrow \underline{\mathcal{E}}$ be the automorphism induced by $F$ on $\underline{\mathcal{E}}$. The following is a key result to prove our main theorem. 
Theorem 32. Suppose that $\underline{\mathcal{E}} / \underline{F}$ is triangulated with respect to $\left(\mathcal{A c}(\mathcal{P})_{d g}, M_{\widetilde{F}}\right)$. Then there is full and faithful triangulated functor $\underline{\mathcal{E}} / \underline{F} \rightarrow \underline{\operatorname{gpr}}(\mathcal{P} / F)$ which makes the following diagram commutative up to isomorphism

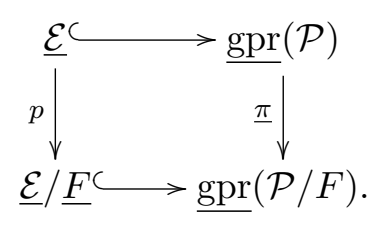

Remark 33. Theorem 31 and Theorem 32 can be stated for completed orbit categories. The statements and the proofs in both settings are essentially the same. In section 6 we will give a proof of these theorems for completed orbit categories $c f$. Corollary 40 and Remark 37. For this reason, we will omit the proofs of these statements.

\section{Completed orbit CAtegories}

Let $k$ be a field and $\mathcal{A}$ an essentially small additive category. Let $F: \mathcal{A} \rightarrow \mathcal{A}$ be an automorphism of $\mathcal{A}$ such that for all objects $X, Y$ of $\mathcal{A}$, the space $\mathcal{A}\left(X, F^{l}(Y)\right)$ vanishes for all integers $l \ll 0$ (whenever we make reference to completed orbit categories we will implicitly assume this condition). We define the completed orbit category $\mathcal{A} \widehat{/ F}$ as the category whose objects are the same as those of $\mathcal{A}$ and with morphism spaces

$$
\mathcal{A} \widehat{/ F}(X, Y)=\prod_{l \in \mathbb{Z}} \mathcal{A}\left(X, F^{l}(Y)\right)
$$

Notice that the vanishing condition imposed on the spaces $\mathcal{A}\left(X, F^{l}(Y)\right)$ ensures that the composition in $\mathcal{A} \widehat{/ F}$ defined as for the usual orbit category in (2.2) is a well-defined operation. Clearly, the category $\mathcal{A} \widehat{/ F}$ is $k$-linear and essentially small. Let $p: \mathcal{A} \rightarrow \mathcal{A} \widehat{/ F}$ be the natural projection. As before, $p$ induces a pair of adjoint functors

$$
\begin{gathered}
\operatorname{Mod}(\mathcal{A}) \\
\pi \downarrow \mid p^{*} \\
\operatorname{Mod}(\mathcal{A} \widehat{/ F}),
\end{gathered}
$$

where $p^{*}$ is the restriction functor and its left adjoint $\pi$ takes a projective module $\mathcal{A}(?, X)$ to the projective module $\mathcal{A} \widehat{/ F}(?, p(X))$. We denote by $F_{*}$ the automorphism $M \mapsto M \circ F^{-1}$ of $\operatorname{Mod}(\mathcal{A})$ induced by $F$.

Lemma 34. Let $M$ be a finitely presented $\mathcal{A}$-module. Then

(i) we have a canonical isomorphism

$$
p^{*} \pi(M) \stackrel{\sim}{\longrightarrow} \prod_{l \in \mathbb{Z}} F_{*}^{l}(M),
$$

(ii) let $L$ be an $\mathcal{A}$-module admitting a resolution $\cdots \rightarrow P_{1} \rightarrow P_{0} \rightarrow L \rightarrow 0$ by finitelygenerated projective $\mathcal{A}$-modules $P_{i}$. Then the complex

$$
\cdots \longrightarrow \pi\left(P_{1}\right) \longrightarrow \pi\left(P_{0}\right) \longrightarrow \pi(L) \longrightarrow 0
$$

is a resolution of $\pi(L)$ by finitely-generated projective $\mathcal{A} \widehat{/ F}$-modules, 
(iii) for each $\mathcal{A}$-module $L$ admitting a resolution by finitely-generated projective $\mathcal{A}$-modules, there are canonical isomorphisms

$$
\operatorname{Ext}_{\operatorname{Mod}(\mathcal{A} \widehat{F F})}^{i}(\pi(L), \pi(M)) \cong \prod_{l \in \mathbb{Z}} \operatorname{Ext}_{\operatorname{Mod}(\mathcal{A})}^{i}\left(L, F_{*}^{l}(M)\right)
$$

for all $i \geq 0$,

(iv) if $\mathcal{E}$ is an exact subcategory of $\bmod (\mathcal{A})$ stable under the action of $F$, then we have a square commutative up to isomorphism

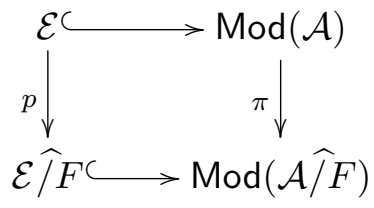

with fully faithful horizontal arrows.

Proof. (i) Since $\pi(\mathcal{A}(?, X))=\mathcal{A} \widehat{/ F}(?, p(X))=\prod_{l \in \mathbb{Z}} F_{*}^{l}(\mathcal{A}(?, X))$, we have that $p^{*} \pi(P)=$ $\prod_{l \in \mathbb{Z}} F_{*}^{l}(P)$ for all projective modules of finite type. Since $p^{*} \pi$ is right exact, we have

$$
p^{*} \pi(M)=\prod_{l \in \mathbb{Z}} F_{*}^{l}(M)
$$

for all $M$ in $\bmod (\mathcal{A})$.

(ii) By $(i)$ the complex $\cdots \rightarrow p^{*} \pi\left(P_{1}\right) \rightarrow p^{*} \pi\left(P_{0}\right) \rightarrow p^{*} \pi(L) \rightarrow 0$ is exact and for all $l \geq 0, \pi\left(P_{l}\right)$ is a finitely-generated projective $\mathcal{A} \widehat{/ F}$-module. Since $p^{*}$ is the restriction functor, the claim follows.

(iii) We have the following isomorphisms

$$
\begin{aligned}
\operatorname{Hom}_{\mathcal{A} \widehat{T F}}(\pi(L), \pi(M)) & \cong \operatorname{Hom}_{\mathcal{A}}\left(L, p^{*} \pi(M)\right) \\
& \cong \operatorname{Hom}_{\mathcal{A}}\left(L, \prod_{l \in \mathbb{Z}} F_{*}^{l}(M)\right) \\
& \cong \prod_{l \in \mathbb{Z}} \operatorname{Hom}_{\mathcal{A}}\left(L, F_{*}^{l}(M)\right) .
\end{aligned}
$$

By $(i i)$, the complex $\cdots \rightarrow \pi\left(P_{1}\right) \rightarrow \pi\left(P_{0}\right) \rightarrow \pi L \rightarrow 0$ is a projective resolution of $\pi(L)$. After applying the functor $\operatorname{Hom}_{\mathcal{A} \widehat{F F}}(?, \pi(M))$ to this resolution and the last isomorphism in (4.3) the claim follows.

(iv) This is immediate from (4.3).

Lemma 35. (i) If $X$ is an object of $\mathcal{A}$ such that $\mathcal{A}(X, X)$ is local and $\mathcal{A}\left(X, F^{l}(X)\right)$ vanishes for all $l<0$, then $\mathcal{A} \widehat{/ F}(p(X), p(X))$ is local.

(ii) If $\mathcal{A}$ is a Krull-Schmidt category such that for each indecomposable object $X$, the ring $\mathcal{A}(X, X)$ is local and $\mathcal{A}\left(X, F^{l}(X)\right)$ vanishes for all $l<0$, then $\mathcal{A} \widehat{/ F}$ is a Krull-Schmidt category whose indecomposables are the images of those of $\mathcal{A}$.

Proof. (i) We can easily see that $\left(f_{i}\right) \in \mathcal{A} \widehat{/ F}(p(X), p(X))$ is non-invertible if and only if $f_{0}: X \rightarrow X$ is non-invertible. This shows that $\mathcal{A} \widehat{/ F}(p(X), p(X))$ is local.

(ii) By part $(i)$, the image $p(X)$ of each indecomposable $X$ of $\mathcal{A}$ is indecomposable with local endomorphism ring. Hence, since each object of $\mathcal{A}$ decomposes into a sum of indecomposables, the same holds for $\mathcal{A} \widehat{/ F}$.

Suppose that $\mathcal{A}$ has enough projectives and let $\mathcal{P}$ denote its full subcategory of projective objects. The essential image of $\mathcal{A}$ under $p$ is canonically identified with $\mathcal{P} \widehat{/ F}$. Let $\langle\mathcal{P} \widehat{/ F}\rangle$ be 
the ideal of morphisms of $\mathcal{A} \widehat{/ F}$ which factor through $\mathcal{P} \widehat{/ F}$. Denote by $\underline{F}$ the automorphism in $\underline{\mathcal{A}}$ induced by $F$. The canonical projection $p: \mathcal{A} \rightarrow \mathcal{A} \widehat{/ F}$ induces an $\underline{F}$-invariant functor $\mathcal{A} \longrightarrow \mathcal{A} \widehat{/ F} /\langle\mathcal{P} \widehat{/ F}\rangle$. By the universal property of orbit categories we obtain a functor $\psi: \underline{\mathcal{E}} / \underline{F} \longrightarrow \mathcal{E} \widehat{\mathcal{F}} F /\langle\mathcal{P} \widehat{/ F}\rangle$.

Proposition 36. The functor $\psi$ is faithful. Moreover, if for all objects $X, Y$ of $\mathcal{A}$ we have that $\underline{\mathcal{A}}\left(X, \underline{F}^{l}(Y)\right)=0$ for $l \gg 0$, then $\psi$ is fully.

Proof. For simplicity, along the proof we will denote $\mathcal{A} \widehat{/ F} /\langle\mathcal{P} \widehat{/ F}\rangle$ by $\mathcal{A} \widehat{\mathcal{F} F}$. Let $P \stackrel{f}{\rightarrow} Y$ be a projective cover in $\mathcal{A}$. Then the morphism

$$
F^{l}(P) \stackrel{F^{l}(f)}{\longrightarrow} F^{l}(Y)
$$

is a projective cover for all $l \in \mathbb{Z}$. In particular, every morphism $p\left(P^{\prime}\right) \rightarrow p(Y)$ in $\mathcal{A} \widehat{/ F}$ with $P^{\prime} \in \mathcal{P}$ can be factorized through $p(f)$. Moreover, since $p(f)$ is concentrated in one degree we obtain the following isomorphisms

$$
\begin{aligned}
& \underline{\mathcal{A} \widehat{/ F}(p(X), p(Y))} \cong \operatorname{cok}(\mathcal{A} \widehat{/ F}(p(X), p(P)) \rightarrow \mathcal{A} \widehat{/ F}(p(X), p(Y))) \\
& \cong \operatorname{cok}\left(\prod_{l \in \mathbb{Z}} \mathcal{A}\left(X, F^{l}(P)\right) \rightarrow \prod_{l \in \mathbb{Z}} \mathcal{A}\left(X, F^{l}(Y)\right)\right) \\
& \cong \prod_{l \in \mathbb{Z}}\left(\operatorname{cok}\left(\mathcal{A}\left(X, F^{l}(P)\right) \rightarrow \mathcal{A}\left(X, F^{l}(Y)\right)\right)\right. \\
& \cong \prod_{l \in \mathbb{Z}} \underline{\mathcal{A}}\left(X, \underline{F}^{l}(Y)\right) \\
&=\underline{\mathcal{A}} \widehat{\widehat{F}}(X, Y) .
\end{aligned}
$$

We consider $\underline{\mathcal{A}} / \underline{F}$ as a subcategory of $\underline{\mathcal{A}} \widehat{\widehat{F}}$. One checks that, up to equivalence, the functor $\mathcal{A} / \underline{F} \rightarrow \widehat{\mathcal{A} / F}$ is given by this chain of isomorphisms at the level of morphisms. Both claims follow.

Remark 37. Lemma 34 and Proposition 36 can be stated for usual orbit categories. The statements and the proofs are essentially the same. Notice that for usual orbit categories the functor $\psi: \underline{\mathcal{A}} / \underline{F} \longrightarrow \mathcal{A} / F /\langle\mathcal{P} / F\rangle$ is always full and faithful.

\section{Comparison of the triangulated structures}

We use the universal property of dg orbit categories to construct a triangulated functor which will be crucial in the proof of the main theorem. Let $\mathcal{E}$ be a Frobenius category endowed with an exact automorphism $F: \mathcal{E} \rightarrow \mathcal{E}$. The restriction of $F$ to $\mathcal{P}$ induces an automorphism of $\mathcal{P}$. We denote by $\widetilde{F}: \mathcal{A} c(\mathcal{P})_{d g} \rightarrow \mathcal{A} c(\mathcal{P})_{d g}$ the dg functor given by $F$ componentwise. Notice that $M_{\widetilde{F}}$ induces a triangle functor on $\underline{\mathcal{E}}$ which is equivalent to $\underline{F}$. We identify $\mathcal{E}$ with an exact subcategory of $\operatorname{Mod}(\mathcal{P})$ via the restricted Yoneda embedding. The adjoint functors $\pi: \operatorname{Mod}(\mathcal{P}) \rightleftarrows \operatorname{Mod}(\mathcal{P} \widehat{/ F}): p^{*}$ induce a pair of adjoint functors

$$
\begin{gathered}
\mathcal{C}_{d g}(\mathcal{P}) \\
\tilde{\pi}_{\Downarrow} \mid \hat{p}^{*} \\
\mathcal{C}_{d g}(\mathcal{P} \widehat{/ F}) .
\end{gathered}
$$


defined componentwise. We consider $\mathcal{A} c(\operatorname{proj}(\mathcal{P}))_{d g}\left(\operatorname{resp} . \mathcal{A} c(\operatorname{proj}(\mathcal{P} \widehat{/ F}))_{d g}\right)$ as a full subcategory of $\mathcal{C}_{d g}(\mathcal{P})\left(\right.$ resp. $\left.\mathcal{C}_{d g}(\mathcal{P} \widehat{/ F})\right)$, see Example 9 and Notation 24 .

Lemma 38. The functor $\tilde{\pi}$ restricts to a functor

$$
\tilde{\pi}: \mathcal{A} c(\operatorname{proj}(\mathcal{P}))_{d g} \rightarrow \mathcal{A} c(\operatorname{proj}(\mathcal{P} \widehat{/ F}))_{d g} .
$$

Proof. Let $P^{\cdot}: \cdots \rightarrow P_{-1} \rightarrow P_{0} \rightarrow P_{1} \rightarrow \cdots$ be a complex in $\mathcal{A} c(\operatorname{proj}(\mathcal{P}))_{d g}$. Then the complex $\tilde{\pi}\left(P^{\cdot}\right)=\cdots \rightarrow \pi\left(P_{1}\right) \rightarrow \pi\left(P_{0}\right) \rightarrow \pi\left(P_{-1}\right) \rightarrow \cdots$ is a complex of finitely-generated projective $\mathcal{P} \widehat{F} F$-modules. For each $i \in \mathbb{Z}$ consider the truncated complex

$$
\cdots \rightarrow \pi\left(P_{i-1}\right) \rightarrow \pi\left(P_{i}\right) \rightarrow M \rightarrow 0
$$

i.e. $M=\operatorname{cok}\left(\pi\left(P_{i-1}\right) \rightarrow \pi\left(P_{i}\right)\right)$. It follows from Lemma 34 that this complex is acyclic. Since $i \in \mathbb{Z}$ is arbitrary then $\pi\left(P^{\cdot}\right)$ is acyclic.

Lemma 39. The functor $\pi: \operatorname{Mod}(\mathcal{P}) \rightarrow \operatorname{Mod}(\mathcal{P} \widehat{/ F})$ restricts to a functor $\pi: \operatorname{gpr}(\mathcal{P}) \rightarrow$ $\operatorname{gpr}(\mathcal{P} \widehat{/ F})$.

Proof. Let $M$ be a module of $\operatorname{gpr}(\mathcal{P})$. In particular, there are objects $\left(P_{i}\right)_{i \in \mathbb{Z}}$ of $\mathcal{P}$ and an acyclic complex

$$
P^{\cdot}: \cdots \rightarrow \mathcal{P}\left(?, P_{i}\right) \rightarrow \mathcal{P}\left(?, P_{i-1}\right) \rightarrow \cdots
$$

such that $M \cong Z^{0}\left(P^{\cdot}\right)$. By Lemma 38 , the complex

$$
\tilde{\pi}\left(P^{\cdot}\right)=\cdots \rightarrow \mathcal{P}\left(?, p\left(P_{i}\right)\right) \rightarrow \mathcal{P}\left(?, p\left(P_{i-1}\right)\right) \rightarrow \cdots
$$

is acyclic and $\pi(M) \cong Z^{0}\left(\pi\left(P^{\cdot}\right)\right)$. If $X$ and $Y$ are arbitrary objects of $\mathcal{P}$ then, by part $(i)$ of Lemma 34 and the Yoneda lemma, we obtain an isomorphism

$$
\operatorname{Hom}(\mathcal{P} \widehat{/ F}(?, p(X)), \mathcal{P} \widehat{/ F}(?, p(Y))) \cong \prod_{i} \mathcal{P}\left(X, F_{*}^{l}(Y)\right)
$$

It follows that $\operatorname{Hom}\left(\pi\left(P^{\cdot}\right), P^{\prime}\right)$ is still acyclic for each module $P^{\prime}$ in $\operatorname{proj}(\mathcal{P} \widehat{/ F})$.

The functor $\pi: \operatorname{gpr}(\mathcal{P}) \rightarrow \operatorname{gpr}(\mathcal{P} \widehat{/ F})$ preserves projectives, therefore it induces a functor $\underline{\pi}: \underline{\operatorname{gpr}}(\mathcal{P}) \rightarrow \underline{\operatorname{gpr}}(\mathcal{P} \widehat{/ F})$.

Corollary 40. There is a square commutative up to isomorphism

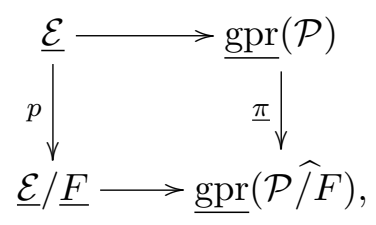

with faithful horizontal arrows. If for all $X$ and $Y$ we have that $\underline{\mathcal{E}}\left(X, F^{l}(Y)\right)=0$ for $l \gg 0$, then $\underline{\mathcal{E}} / \underline{F} \rightarrow \underline{\operatorname{gpr}}(\mathcal{P} \widehat{/ F})$ is fully faithful.

Proof. By Lemma 34 and Lemma 39 there is a commutative diagram

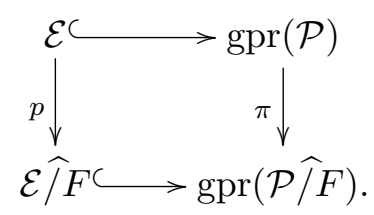


with faithful horizontal arrows. The functor $\mathcal{E} \widehat{/ F} \hookrightarrow \operatorname{gpr}(\mathcal{P} \widehat{/ F})$ identifies $\mathcal{P} \widehat{/ F}$ with $\operatorname{proj}(\mathcal{P} \widehat{/ F})$. Therefore there is a commutative diagram

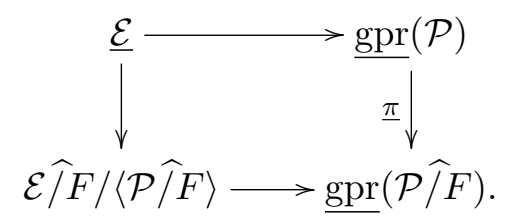

The claim follows from Proposition 36.

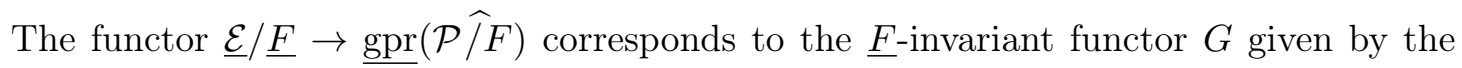
composition

We denote by $\widetilde{G}$ the composition

$$
G: \underline{\mathcal{E}} \hookrightarrow \underline{\operatorname{gpr}}(\mathcal{P}) \rightarrow \underline{\operatorname{gpr}}(\mathcal{P} \widehat{/ F}) .
$$

$$
\widetilde{G}: \mathcal{A} c(\mathcal{P})_{d g} \longrightarrow \mathcal{A} c(\operatorname{proj}(\mathcal{P}))_{d g} \stackrel{\tilde{\pi}}{\longrightarrow} \mathcal{A} c(\operatorname{proj}(\mathcal{P} \widehat{F} F))_{d g}
$$

where the first arrow is given by applying the Yoneda functor $\mathcal{P} \hookrightarrow \operatorname{proj}(\mathcal{P})$ componentwise. Notice that $\mathcal{A} c(\operatorname{proj}(\mathcal{P}))_{d g}$ is a dg enhancement of $\underline{\operatorname{gpr}}(\mathcal{P})$ and that $H^{0}(\widetilde{G})=G$.

Theorem 41. Suppose that $\underline{\mathcal{E}} / \underline{F}$ is triangulated with respect to $\left(\mathcal{A c}(\mathcal{P})_{d g}, M_{\widetilde{F}}\right)$. Then the functor $\underline{\mathcal{E}} / \underline{F} \rightarrow \underline{\operatorname{gpr}}(\mathcal{P} \widehat{/ F})$ is triangulated.

Proof. It is enough to prove that there is a quasi-isomorphism of quasi-functors $\phi: \widetilde{G} \rightarrow$ $\widetilde{G} M_{\widetilde{F}}$ such that $(\widetilde{G}, \phi) \in \operatorname{eff}\left(\mathcal{A} c(\mathcal{P})_{d g}, M_{\widetilde{F}}, \mathcal{A} c(\operatorname{proj}(\mathcal{P} \widehat{/ F}))\right.$. In other words, we have to prove that for all complexes $P^{\cdot} \in \mathcal{A} c(\mathcal{P})_{d g}$ and $Q^{\cdot} \in \mathcal{A} c(\operatorname{proj}(\mathcal{P} \widehat{F} F))_{d g}$, there is a quasiisomorphism

$$
\mathcal{H o m}\left(Q^{\cdot}, \widetilde{G}\left(P^{\cdot}\right)\right) \longrightarrow \mathcal{H o m}\left(Q \cdot \widetilde{G} \widetilde{F}\left(P^{\cdot}\right)\right) .
$$

The complex $P$ is of the form

$$
\cdots \longrightarrow P_{i} \longrightarrow P_{i+1} \longrightarrow \cdots
$$

where $P_{i}$ is object in $\mathcal{P}$ for each $i \in \mathbb{Z}$. Then the complex $\widetilde{G}\left(P^{\cdot}\right)$ is given by

$$
\cdots \longrightarrow \mathcal{P} \widehat{/ F}\left(?, p\left(P_{i}\right)\right) \longrightarrow \mathcal{P} \widehat{/ F}\left(?, p\left(P_{i+1}\right)\right) \longrightarrow \cdots
$$

and the complex $\widetilde{G} \widetilde{F}\left(P^{\cdot}\right)$ is given by

$$
\cdots \longrightarrow \mathcal{P} \widehat{/ F}\left(?, p F\left(P_{i}\right)\right) \longrightarrow \mathcal{P} \widehat{/ F}\left(?, p F\left(P_{i+1}\right)\right) \longrightarrow \cdots .
$$

For each $i \in \mathbb{Z}$ the objects $P_{i}$ and $F\left(P_{i}\right)$ are isomorphic in $\mathcal{P} \widehat{/ F}$. Therefore there is an $\underset{\widetilde{G}}{\operatorname{isomorphism}} \mathcal{P} \widehat{/ F}\left(?, p\left(P_{i}\right)\right) \rightarrow \mathcal{P} \widehat{/ F}\left(?, p F\left(P_{i}\right)\right)$. In particular, the complexes $\widetilde{G}\left(P^{\cdot}\right)$ and $\widetilde{G} \widetilde{F}\left(P^{\cdot}\right)$ are isomorphic. The claim follows.

\section{THE MAIN THEOREMS}

We keep the notation of the preceding section and suppose that $\underline{\mathcal{E}} / \underline{F}$ is equivalent to its triangulated hull with respect to $\left(\mathcal{A} c(\mathcal{P})_{d g}, M_{\widetilde{F}}\right)$.

Theorem 42. Suppose that $\mathcal{E}$ is Krull-Schmidt such that

- for each indecomposable object $X$, the ring $\mathcal{E}(X, X)$ is local and $\mathcal{E}\left(X, F^{l}(X)\right)$ vanishes for all $l<0$,

- for every pair of objects $Y$ and $Z$, the space $\underline{\mathcal{E}}\left(Y, \underline{F}^{l}(Z)\right)$ vanishes for $l \gg 0$. 
Then $\mathcal{E} \widehat{/ F}$ admits the structure of a Krull-Schmidt Frobenius category which makes the canonical projection $\mathcal{E} \rightarrow \mathcal{E} \widehat{/ F}$ exact and whose stable category is triangle equivalent to $\underline{\mathcal{E}} / \underline{F}$.

Proof. By part (iv) of Lemma 34, Lemma 39 and Corollary 40 there is a commutative diagram

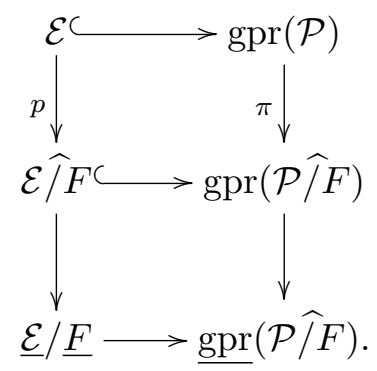

The first two horizontal arrows are full and faithful. So we may think of $\mathcal{E}$ (resp. $\mathcal{E} / F$ ) as a full subcategory of $\operatorname{gpr}(\mathcal{P})($ resp. $\operatorname{gpr}(\mathcal{P} \widehat{/ F}))$. In particular, we identify $p$ with the restriction of $\pi$ to $\mathcal{E}$. We first show that $\mathcal{E} \widehat{/ F}$ is an extension-closed subcategory of $\operatorname{gpr}(\mathcal{P} \widehat{/ F})$. Let $0 \rightarrow \pi(X) \rightarrow E \rightarrow \pi(Y) \rightarrow 0$ be an extension in $\operatorname{gpr}(\mathcal{P} \widehat{/ F})$ between two objects of $\mathcal{E} \widehat{/ F}$. By part (iii) of Lemma 34, we have an isomorphism

$$
\begin{aligned}
\operatorname{Ext}_{\operatorname{gpr}(\mathcal{P} \widehat{T} F)}^{1}(\pi(Y), \pi(X)) & \cong \prod_{l \in \mathbb{Z}} \operatorname{Ext}_{\operatorname{gpr}(\mathcal{P})}^{1}\left(Y, F_{*}^{l}(X)\right) \\
& \cong \prod_{l \in \mathbb{Z}} \operatorname{Ext}_{\mathcal{E}}^{1}\left(Y, F^{l}(X)\right) .
\end{aligned}
$$

Since $\underline{\mathcal{E}}\left(X, \underline{F}^{l}(Y)\right)=0$ for $l \gg 0$ the categories $\underline{\mathcal{E}} \widehat{\widehat{F}} \underline{F}$ and $\underline{\mathcal{E}} / \underline{F}$ are isomorphic. In particular, there is an isomorphisms $\prod_{l \in \mathbb{Z}} \operatorname{Ext}_{\mathcal{E}}^{1}\left(Y, F^{l}(X)\right) \cong \operatorname{Ext}_{\underline{\mathcal{E}} / \underline{F}}^{1}\left(Y, F^{l}(X)\right)$ and therefore, an isomorphism

$$
\operatorname{Ext}_{\operatorname{gpr}(\mathcal{P} / F)}^{1}(\pi(Y), \pi(X)) \cong \operatorname{Ext}_{\underline{\mathcal{E}} / \underline{F}}^{1}(Y, X)
$$

Let

$$
X \longrightarrow E^{\prime} \longrightarrow Y \longrightarrow \Sigma X
$$

be the triangle in $\underline{\mathcal{E}} / \underline{F}$ corresponding to the extension $0 \rightarrow \pi(X) \rightarrow E \rightarrow \pi(Y) \rightarrow 0$ under (6.1). By Theorem 41, there is a commutative diagram in $\underline{\operatorname{gpr}}(\mathcal{P} \widehat{/ F})$

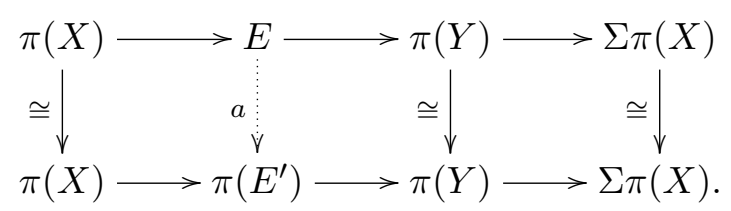

The first row corresponds to the triangle associated to the extension $0 \rightarrow \pi(X) \rightarrow E \rightarrow$ $\pi(Y) \rightarrow 0$, the second row is the image of the triangle $X \rightarrow E^{\prime} \rightarrow Y \rightarrow \Sigma X$ under the triangle functor $\underline{\mathcal{E}} / \underline{F} \rightarrow \underline{\operatorname{gpr}}(\mathcal{P} \widehat{/ F})$. Notice that the square on the right commutes by the choice of $X \rightarrow E^{\prime} \rightarrow Y \rightarrow \Sigma X$. Therefore we obtain the arrow $E \stackrel{a}{\rightarrow} \pi\left(E^{\prime}\right)$ by the axiom of triangulated categories. The Yoneda functor sends each of the rows of this diagram to a long exact sequence (to the left). We apply the five lemma to conclude that $a$ is an isomorphism. Thus, $\pi(E)$ is a direct factor of the sum of $E^{\prime}$ with a finitely-generated projective module. 
By Lemma 35 , we have that $\mathcal{E} \widehat{/ F}$ is Krull-Schmidt. In particular, it has split idempotents and it follows that $\pi(E)$ lies in $\mathcal{E} \widehat{F} F$. Hence $\mathcal{E} \widehat{/ F}$ is stable under extensions in $\operatorname{gpr}(\mathcal{P} \widehat{/ F})$. Notice that the projective-injective objects of $\operatorname{gpr}(\mathcal{P} \widehat{/ F})$ belong to $\mathcal{E} \widehat{/ F}$ and are precisely the image of the projectives of $\mathcal{E}$ under the right exact functor $\pi$. Therefore $\mathcal{E} \widehat{/ F}$ is an exact category with enough projectives and such that projectives are injectives. Since $\mathcal{E} \widehat{\mathcal{F}} F$ is closed under $\Sigma$ in $\operatorname{gpr}(\mathcal{P} \widehat{\mathcal{F}} F)$ it also has enough injectives. Now it is easy to see that $\underline{\mathcal{E} / F}$ is triangle equivalent to $\underline{\mathcal{E}} / \underline{F}$. The exactness of the canonical functor $\mathcal{E} \rightarrow \widehat{\mathcal{E} / F}$ follows from the fact that the induced functor $\underline{\mathcal{E}} \rightarrow \underline{\mathcal{E}} / \underline{F}$ is triangulated.

We can use Theorem 31, Theorem 32 and Remark 37 to prove the following theorem. The proof is essentially the same as the proof of Theorem 42 .

Theorem 43. Suppose that $\mathcal{E} / F$ has split idempotents. Then $\mathcal{E} / F$ admits the structure of a Frobenius category which makes the canonical projection $\mathcal{E} \rightarrow \mathcal{E} / F$ exact and whose stable category is triangle equivalent to $\underline{\mathcal{E}} / \underline{F}$.

Remark 44. If we want to use the results in [21] to determine if $\underline{\mathcal{E}} / \underline{F}$ is triangulated we may assume that $\mathcal{E}$ is Ext-finite, since Keller considers Hom-finite triangulated categories.

Remark 45. We don not have a criterion to determine if $\mathcal{E} / F$ has split idempotents.

\section{Applications to Cluster algebras (Sketch)}

In this section we use the theory of Nakajima categories introduced in [24]. The reader is referred to this article for some of the definitions and relevant background. We can use Theorem 42 to obtain explicit categorifications of families of finite-type cluster algebras with coefficients. In particular, we obtain a categorification of all skew-symmetric finitetype cluster algebras with universal coefficients.

7.1. Nakajima categories. Let $Q$ be a finite and acyclic quiver. The repetition quiver (cf. [31]) $\mathbb{Z} Q$ is the quiver obtained from $Q$ as follows

- the set of vertices of $\mathbb{Z} Q$ is $(\mathbb{Z} Q)_{0}=Q_{0} \times \mathbb{Z}$.

- For each arrow $\alpha: i \longrightarrow j$ of $Q$ and each $p \in \mathbb{Z}, \mathbb{Z} Q$ has the arrows

$$
(\alpha, p):(i, p) \longrightarrow(j, p) \quad \text { and } \quad \sigma(\alpha, p):(j, p-1) \longrightarrow(i, p) .
$$

- $\mathbb{Z} Q$ has no more arrows than the ones described above.

There is a bijection on the set of arrows $\sigma:(\mathbb{Z} Q)_{1} \rightarrow(\mathbb{Z} Q)_{1}$ given by

$$
\sigma(\beta)= \begin{cases}\sigma(\alpha, p) & \text { if } \beta=(\alpha, p), \\ (\alpha, p-1) & \text { if } \beta=\sigma(\alpha, p) .\end{cases}
$$

Let $\tau: \mathbb{Z} Q \rightarrow \mathbb{Z} Q$ be the automorphism of $\mathbb{Z} Q$ given by the translation by one unit:

$$
\tau(i, p)=(i, p-1) \quad \text { and } \quad \tau(\beta)=\sigma^{2}(\beta)
$$

for each vertex $(i, p)$ and each arrow $\beta$ of $\mathbb{Z} Q$.

Let $k$ be a field. Following [14] and [30], we define the mesh category $k(\mathbb{Z} Q)$ to be the quotient of the path category $k \mathbb{Z} Q$ by the ideal generated by the mesh relators, i.e. the $k$-category whose objects are the vertices of $\mathbb{Z} Q$ and whose morphism space from $a$ to $b$ is 
the space of all $k$-linear combinations of paths from $a$ to $b$ modulo the subspace spanned by all elements $u r_{x} v$, where $u$ and $v$ are paths and

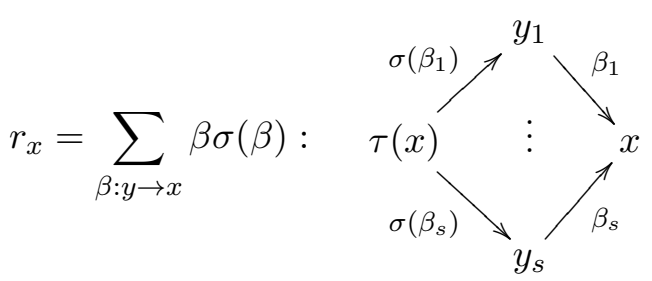

is the mesh relator associated with a vertex $x$ of $\mathbb{Z} Q$. Here the sum runs over all arrows $\beta: y \rightarrow x$ of $\mathbb{Z} Q$.

Definition 46. The framed quiver $\tilde{Q}$ associated to $Q$ is the quiver obtained from $Q$ by adding, for each vertex $i$, a new vertex $i^{\prime}$ and a new arrow $i \rightarrow i^{\prime}$. We call the vertices in $(\mathbb{Z} \tilde{Q})_{0}$ of the form $\left(i^{\prime}, n\right), i \in Q_{0}, n \in \mathbb{Z}$, frozen vertices. The regular Nakajima category $\mathcal{R}$ associated to $Q$ is the quotient of the path category $k \mathbb{Z} \tilde{Q}$ by the ideal generated by the mesh relators associated to the non-frozen vertices. The singular Nakajima category $\mathcal{S}$ is the full subcategory of $\mathcal{R}$ whose objects are the frozen vertices.

Remark 47. Note that there is a bijection $\sigma:(\mathbb{Z} \tilde{Q})_{0} \rightarrow(\mathbb{Z} \tilde{Q})_{0}$ given by $\sigma:(i, n) \mapsto$ $\left(i^{\prime}, n-1\right)$ and $\left(i^{\prime}, n\right) \mapsto(i, n)$ for $i$ a vertex of $Q$ and $n$ an integer.

Assumption 48. From now on we let $Q$ be an orientation of a simply laced Dynkin diagram $\Delta$ and denote the bounded derived category of $\bmod (k Q)$ by $\mathcal{D}_{Q}^{b}$.

Definition 49. Let $C$ be a subset of $(\mathbb{Z} Q)_{0}$. Denote by $\mathcal{R}_{C}$ the quotient of $\mathcal{R}$ by the ideal generated by the identities of the frozen vertices not belonging to $\sigma^{-1}(C)$ and by $\mathcal{S}_{C}$ its full subcategory formed by the vertices in $\sigma^{-1}(C)$. We call $C$ an admissible configuration of $\mathbb{Z} Q$ if the sequences

$$
0 \rightarrow \mathcal{R}_{C}(?, x) \rightarrow \bigoplus_{x \rightarrow y} \mathcal{R}_{C}(?, y) \quad \text { and } \quad 0 \rightarrow \mathcal{R}_{C}(x, ?) \rightarrow \bigoplus_{y \rightarrow x} \mathcal{R}_{C}(y, ?)
$$

are exact, where the sums range over all arrows of $\mathbb{Z} \tilde{Q}$ whose source (respectively, target) is $x$. We denote by $\mathbb{Z} \tilde{Q}_{C}$ the quiver obtained from $\mathbb{Z} \tilde{Q}$ by removing the vertices not belonging to $\sigma^{-1}(C)$. We refer the reader to Section 3.3 of [24] for an account of sufficient conditions on $C$ in which (7.1) holds.

Theorem 50. ([24]) Let $C$ be an admissible configuration of $\mathbb{Z} Q$. Then

(i) the restriction functor $\operatorname{Mod} \mathcal{R}_{C} \rightarrow \operatorname{Mod} \mathcal{S}_{C}$ induces an equivalence between the full subcategory of finitely generated projective $\mathcal{R}_{C}$-modules $\operatorname{proj}\left(\mathcal{R}_{C}\right)$ and the category $\operatorname{gpr}\left(\mathcal{S}_{C}\right)$. In particular, it yields an isomorphism of $\mathbb{Z} \tilde{Q}_{C}$ onto the AuslanderReiten quiver of $\operatorname{gpr}\left(\mathcal{S}_{C}\right)$ so that the vertices of $\sigma^{-1}(C)$ correspond to the projectiveinjective objects,

(ii) there is a triangle equivalence $\Phi: \underline{\operatorname{gpr}}\left(\mathcal{S}_{C}\right) \rightarrow \mathcal{D}_{Q}^{b}$.

7.2. Categorification of cluster algebras with coefficients. Let $\tau: \mathcal{D}_{Q}^{b} \rightarrow \mathcal{D}_{Q}^{b}$ be the Auslander-Reiten translation and $\Sigma: \mathcal{D}_{Q}^{b} \rightarrow \mathcal{D}_{Q}^{b}$ be the suspension functor of $\mathcal{D}_{Q}^{b}$. The cluster category $\mathcal{C}_{Q}$ was introduced in [6] and is defined as the orbit category

$$
\mathcal{C}_{Q}=\mathcal{D}_{Q}^{b} / \Sigma \circ \tau^{-1} .
$$

The category $\mathcal{C}_{Q}$ is triangulated. Its triangulated structure comes from the dg category $C^{b}(\operatorname{proj} k Q) d g$. 
We denote $\Sigma \circ \tau^{-1}$ by $F_{\mathcal{D}}$. By a well know result of Happel [15], we know that the Auslander-Reiten quiver of the category $\mathcal{D}_{Q}^{b}$ is canonically isomorphic to $\mathbb{Z} Q$. Therefore, $F_{\mathcal{D}}$ induces an automorphism of translation quivers $F: \mathbb{Z} Q \rightarrow \mathbb{Z} Q$. Let $C \subset \mathbb{Z} Q$ be an admissible configuration which is invariant under $F$. By Theorem $50, F$ induces a functor

$$
F_{*}: \operatorname{gpr}\left(\mathcal{S}_{C}\right) \rightarrow \operatorname{gpr}\left(\mathcal{S}_{C}\right)
$$

which is in fact exact. Theorem 42 is fundamental to prove the following results.

Lemma 51. The completed orbit category $\operatorname{gpr}(\mathcal{S}) \widehat{/ F_{*}}$ admits the structure of a Frobenius category whose stable category is triangle equivalent to $\mathcal{C}_{Q}$.

Theorem 52. (i) Let $C \subset \mathbb{Z} Q$ be an admissible configuration which is invariant under $F$. Then $\operatorname{gpr}\left(\mathcal{S}_{C}\right) \widehat{/ F}$ is a 2-Calabi-Yau realization (in the sense of [5]) of a cluster algebra with coefficients of type $\Delta$,

(ii) if $C=\mathbb{Z} Q$ then $\operatorname{gpr}\left(\mathcal{S}_{C}\right) \widehat{/ F}$ is a 2-Calabi-Yau realization of the cluster algebra with universal coefficients of type $\Delta$.

The proofs of Lemma 51 and Theorem 52, the details of this section and further applications will be given in a forthcoming paper [27].

\section{REFERENCES}

[1] H. Asashiba, The 2-categories of G-categories and of G-graded categories are 2-equivalent, arXiv:0905.3884.

[2] __, A generalization of Gabriel's Galois covering functors and derived equivalences, Journal of Algebra 334 (2011), No. 1, 109-149.

[3] M. Auslander and I. Reiten, Applications of contravariantly finite subcategories. Adv. Math. 86 (1991), $111-152$.

[4] A. I. Bondal and M. M. Kapranov, Enhanced triangulated categories, Mat. Sb. 181 (1990), no. 5, 669-683, translation in Math. USSR-Sb. 70 no. 1, 93-107.

[5] A. B. Buan, O. Iyama, I. Reiten, J. Scott, Cluster structures for 2-Calabi-Yau categories and unipotent groups, Amer. J. Math. 133 (2011), no. 4, 835-887.

[6] A. B. Buan, R. J. Marsh, M. Reineke, I. Reiten and G. Todorov, Tilting theory and cluster combinatorics, Advances in Mathematics 204 (2) (2006), 572-618.

[7] T. Bühler, Exact categories. Expo. Math. 28 (2010), 1-69.

[8] X. U. Chen, Three results on Frobenius categories, Mathematische Zeitschrift 270 (2012), 43-58.

[9] C. Cibils and E. Skew category, Galois covering and smash product of a k-category, Proceedings of the AMS 134 (2005), no. 1, 39-50.

[10] V. Drinfeld, DG quotients of DG categories, J. Algebra 272 (2004), no. 2, 643-691.

[11] S. Fomin, A. Zelevinsky, Cluster algebras I: Foundations, J. Amer. Math. Soc. 15 (2002), 497-529.

[12] _ Clusters algebras IV: Coefficients, Composito Mathematica 143 (2007), 112-164.

[13] Ch. Fu, On root categories of finite-dimensional algebras, arXiv:1103.3335.

[14] _ A Aslander-Reiten sequences and representation-finite algebras, Representation theory, I (Proc. Workshop, Carleton Univ., Ottawa, Ont., 1979), Springer, Berlin, 1980, pp. 1-71.

[15] D. Happel, On the derived category of a finite-dimesional algebra, Comment. Math. Helv. 62 (1987), no. $3,339-389$.

[16] - Triangulated categories in the representation theory of finite-dimensional algebras, Cambridge University Press, Cambridge, 1988.

[17] K. Igusa, G. Todorov, Continuous cluster categories I, Algebras and Representation Theory (2014), DOI $10.1007 / \mathrm{s} 10468-014-9481-z$.

[18] B. Keller, Chain complexes and stable categories, Manus. Math. 67 (1990), 379-417.

[19] _ Deriving DG categories, Ann. Scient. Ec. Norm. Sup. 27 (1994), 63-102.

[20] - On differential graded categories, in: International Congress of Mathematicians, vol. II, European Mathematical Society, Zürich, 2006, pp. 151-190. MR2275593 (2008g:18015).

[21] __ On triangulated orbit categories, Doc. Math. 10 (2005), 551-581.

$[22]-$ Corrections to "On triangulated orbit categories", available at the author's webpage. 
[23] Cluster algebras, quiver representations and triangulated categories, Workshop on Triangulated Categories (Leeds, 2006).

[24] B. Keller and S. Scherotzke, Graded quiver varieties and derived categories, J. reine angew. Math., DOI 10.1515 / crelle-2013-0124.

[25] B. Keller and D. Vossieck, Sous les catégories dérivées, C. R. Acad. Sci. Paris, 305 (1987), 225-228.

[26] H. Krause, Functors on locally finitely presented additive categories, Colloquium Mathematicum, 75 (1998), no. 1, 105-132.

[27] A. Nájera Chávez, A 2-Calabi-Yau realization of finite-type cluster algebras with universal coefficients, in preparation.

[28] F. Qin, Quantum groups via cyclic quiver varieties I, arXiv:1312.1101.

[29] D. Quillen, Higher Algebraic K-theory I, Springer LNM 341, 1973, 85-147.

[30] Ch. Riedtmann, Algebren, Darstellungsköcher, Überlagerungen und Zurück, Comment. Math. Helv. 55 (1980), no. 2, 199-224.

[31] _ Representation-finite self-injective algebras of class $A_{n}$, Representation theory, II (Proc. Second Internat. Conf., Carleton Univ., Ottawa, Ont., 1979), Lecture Notes in Math., vol. 832, Springer, Berlin, 1980, pp. 449-520.

[32] S. Scherotzke, Quiver varieties and self-injective algebras, arXiv:1405.4729.

[33] G. Tabuada, Invariants additifs de dg-catégories, Int. Math. Res. Notices 53 (2005), 3309-3339.

Max-Planck-Institut Für Mathematik, Vivatsgasse 7, 53111 Bonn, Germany.

E-mail address: alfredo.najera-chavez@imj-prg.fr 